\title{
Rückblick und Ausblick: Für eine dekoloniale politische Bildung
}

Während ich diesen Text abschließe, überschlagen sich die Ereignisse. In der Folge rassistischer Morde an Schwarzen Menschen durch Polizist_innen wächst die seit 2013 existierende Black Lives Matter-Bewegung international zu einer Massenbewegung. Auslöser - nicht der Grund, denn rassistische Morde durch Polizist_innen fanden und finden in erschreckender Regelmäßigkeit statt - war der Mord an George Floyd am 25. Mai 2020 in Minneapolis. Auch in Deutschland wird in der Folge gegen strukturellen Rassismus - und hier insbesondere gegen auch in Deutschland stattfindende, rassistische Morde und die hier sichtbar werdende koloniale Ungleichwertigkeit des Lebens - protestiert. Hierzulande und international wird dabei der strukturelle Rassismus in eine historische Kontinuität zur Geschichte von Sklaverei und Kolonialismus gestellt. Angela Davis fasst die dekolonialen Implikationen der Black Lives Matter-Bewegung folgendermaßen zusammen:

\footnotetext{
“The seemingly simple phrase 'Black Lives Matter' has disrupted undisputed assumptions about the logic of equality, justice, and human freedom in the United States and all over the world. It has encouraged us to question the capacity of logic - Western logic - to undo the forces of history, especially the history of colonialism and slavery." (Davis 2017: xiii)
}

In symbolisch wirkmächtigen Bildern wird die Forderung nach einer die Vergangenheit und Gegenwart betreffenden und damit auf eine andere Zukunft zielenden Dekolonisierung sichtbar. Diese Forderung wird zum Ausdruck gebracht, indem beispielsweise die Statuen von Sklavenhändlern - wie die Statue des als ,Philanthrop" der Stadt geehrten Edward Colston in Bristol - oder von Kolonialisten - wie die Statue von Kolumbus in Virginia, Boston und Richmond - von Demonstrierenden in Flüsse geworfen, umgeschmissen oder geköpft wurden. 
Das koloniale Erbe wird durch diese dekolonialen Bewegungen zu einem schwierigen Erbe, da es gegenwärtig hegemoniale Identifikationsprozesse, Subjektivierungsweisen, Legitimationsmuster und sinngebende Narrative nachhaltig unterläuft, indem diese in ihrer Kontinuität zur kolonialen Vergangenheit und Kolonialität der Gegenwart sichtbar gemacht werden. Die Aushandlung des Erbes - wie etwa die aktive Intervention durch das Köpfen der Kolumbus-Statue wird damit in ein wechselseitiges Verhältnis zur Frage der (Un-)Gleichheit in der Gegenwart gestellt, damit zu einem Streit und zu einer Frage des Politischen. Im Sinne von Lara (4.2.4) stellt die Anerkennung des kolonialen Erbes eine Ressource dar, um anders zu denken, neokoloniale Machtverhältnisse und rassistische Positionierungen zu problematisieren, infrage zu stellen und eigene Verstricktheiten zu reflektieren. Dabei gibt es kein feststehende Theoriegebäude, Kanon oder Erbe der Dekolonialität. Dekoloniale politische Bildung kann und muss sich vielmehr auf ein - immer wieder neu zu konstruierendes und zu artikulierendes - dekoloniales Erbe beziehen, also die vielfältigen Formen in der Vergangenheit und Gegenwart, in denen koloniale und neokoloniale Machtverhältnisse unterminiert und infrage gestellt worden sind. Eine Form, dieses Verhältnis zum dekolonialen Erbe zu denken, habe ich anhand einer Re-Lektüre von Aimé Césaire herausgearbeitet (2.3.3.4).

Ich hoffe darauf, dass durch diese Konjunktur der Aufmerksamkeit für die Fragen der (De-)Kolonialität lang anhaltende Prozesse tiefgreifender Veränderung entstehen. Eine Zukunftsprognose für eine solche Bewegung und die gesellschaftlichen Reaktionen ist unhaltbar. In jedem Fall aber stellt die Black Lives Matter-Bewegung nicht zuletzt auch die politische Bildung vor große Herausforderungen. Sich diesen Herausforderungen zu stellen und sich (dekolonial und rassismuskritisch) zu positionieren, liegt meiner Meinung nach in der Verantwortung von politischer Bildung. Die komplexe Aufgabe besteht dabei aus meiner Sicht darin, sich gleichzeitig klar zu positionieren und sich immer wieder selbst zu hinterfragen. Es gibt kein fertiges Programm für dekoloniale Bildung; und noch viel weniger kann der hier vorliegende Text ein solches präsentieren oder sollte aus meiner Sicht danach streben. Ich verstehe meinen Text als Diskussionsbeitrag - nicht mehr, aber auch nicht weniger - der hoffentlich als Teil eines Dialogs dazu beitragen kann, dekoloniale Perspektiven zu stärken.

Ich werde an dieser Stelle nicht zusammenfassend wiederholen, was ich geschrieben habe, sondern lieber darüber schreiben, was ich ausgelassen habe und die Begrenztheit der Perspektive benennen. Ganz offensichtlich ist meine Analyse durch die Auswahl der Untersuchungsgruppe von Schüler_innen aus 9. Klassen begrenzt. Es stellt sich die Frage, die in anderen Untersuchungen zu 
überprüfen wäre, inwiefern ähnliche oder gänzlich andere Ergebnisse beispielsweise bei Schüler_innen der Grundschule oder bei Erwachsenen zu erwarten wäre. Nennenswert ist an dieser Stelle vielleicht, dass eine mögliche, wenn auch nicht von mir vertretene Hypothese, dass Hauptschüler_innen weniger reflektiert über Herrschaft nahdenken würden, sich in meiner Untersuchung nicht bestätigt hätte. Koloniale und dekoloniale Momente sind in den Schüler_innenvorstellungen unabhängig von der Schulform und damit der zu vermutenden sozialen Klassierung in gleichem Maße und in gleichen Formen anzutreffen. Darüber hinaus könnte es sinnvoll sein, auch (angehende) Lehrkräfte zu untersuchen, um eine Anpassung von Aus- und Weiterbildungen besser konturieren zu können. Anhand der Erfahrungen im Erhebungsprozess der Untersuchung hat sich jedenfalls in einigen Fällen ziemlich dramatisch gezeigt, dass Lehrkräfte nicht nur als Teil der Lösung, sondern auch als Teil des Problems angesehen werden müssen.

Rückblickend erscheint es mir so, dass ich gleichzeitig zu viel und zu wenig in meinem Text berïcksichtigt habe. Durch das induktiv-deduktive Vorgehen der Kategorienbildung im Rahmen der qualitativen Inhaltsanalyse hat sich das Themenspektrum meiner Forschung weit über das von mir erwartete Spektrum hinaus vervielfältigt. In der Breite des Themenspektrums liegt die Gefahr, dass ich so einzelne Aspekte zu kursorisch bearbeitet haben könnte. Gleichzeitig kommen aus meiner Sicht substanzielle Dimensionen nur als Randphänomene vor, insbesondere die Geschlechterdimension als verschränkt mit der Kolonialität. Die Genderdimension erfasse ich insbesondere und fast ausschließlich im Kontext meiner Analyse der Schüler_innenvorstellungen von Prozessen des orientalisierenden Othering. Bereits in der Erhebung, also der Durchführung der Interviews, habe ich keinen Schwerpunkt auf die Genderdimension gelegt, sodass auch der Versuch, diese Dimension stärker in die Analyse einzubeziehen, zum Scheitern verurteilt war. Unklar ist mir hier, ob dies meinen unbewussten Schwerpunktsetzungen oder dem Zugang über den Begriff der Globalisierung geschuldet ist. Jedenfalls muss hier aus meiner Sicht in zukünftigen Studien anders vorgegangen werden, sodass diese Dimension eine stärkere Berücksichtigung finden kann. Gerade in Bezug auf die migrationsgesellschaftlichen Aspekte und die Konsequenzen für politische Bildung habe ich zwar vielfältige theoretische Ansätze diskutiert und einbezogen, allerdings die bestehenden empirischen Studien in Bezug auf diese Aspekte zu wenig mit einbezogen. Dieses Defizit ist auch der Breite des von mir behandelten Themenspektrums geschuldet.

Angesichts dieser ,Defizite' oder Lücken, die sich aus der Breite des Themenspektrums ergeben, stellt sich nun die Frage, ob diese Breite des Themenspektrums nicht nur als ,Defizit", sondern möglicherweise auch als Potenzial 
zu sehen ist. Meine Hoffnung war es, mit einer dekolonialen Perspektive verschiedene Themenfelder zusammenzubringen, die sonst oft nur kursorisch in ihrem Zusammenhang diskutiert werden. Diese Idee entwickelte sich insbesondere vor dem Hintergrund der Interviews mit den Schüler_innen, in denen Aspekte der internationalen Arbeitsteilung und der globalen Ungleichheit, des Grenzregimes und der Migration sowie der migrationsgesellschaftlichen Zugehörigkeitsregime und Selfing/Othering-Prozesse als direkt verknüpft und in Bezug aufeinander erscheinen. Für die Schüler_innen erscheinen also Zusammenhänge, die auch in einigen akademischen Ansätzen benannt, selten jedoch theoretisiert und adressiert werden. Dies wird auch in vielen Bildungskontexten deutlich, in denen die genannten Themenfelder sehr selten in ihrer Verschränkung bearbeitet werden. Meine Hoffnung war und ist es, dass diese als getrennt erscheinenden Aspekte durch eine dekoloniale Perspektive zusammengebracht und in ihrer Verschränkung bearbeitbar werden.

Der zentrale Begriff um diese Dimensionen zusammen zu denken, ist der Begriff der (De-)Kolonialität (2.3.3), wie er aus der Perspektive des Kontextes in Deutschland beispielsweise durch Iman Attia und Mariam Popal (2016) diskutiert wurde. Diese meinen mit Kolonialität - in Referenz auf Aníbal Quijano - ,ein Netz von Machtstrukturen und interdependenten ökonomischen, politischen und epistemologischen, global und kolonial wirkenden Formationen, die sich in den westlichen Staaten zu spezifischen Denk- und Handlungsmustern verdichteten“ (Attia/Popal 2016: 652). Sie heben hervor, dass es dieser Ansatz „,deutlicher als einige aktuelle postkoloniale Theorierezeptionen im Westen“ (ebd.) ermöglicht, die Gegenwärtigkeit, die Aktualisierung und die Transformation kolonialer Formationen in jeweils neuen historischen Kontexten und Konstellationen zu benennen und zu beschreiben. Kolonialität wird also nicht als Überbleibsel des historischen Kolonialismus verstanden, sondern als eine gegenwärtige Form der Strukturierung von Welt und Denken.

Dieses Verständnis von Kolonialität geht damit einher, dass einige Formen der postkolonialen Theorierezeption im ,Westen' häufig auf die Veränderung von Repräsentationsverhältnissen fokussieren und dabei die sozioökonomischen Macht- und Ungleichheitsverhältnisse marginalisieren bzw. nicht als Teil ihres analytischen Feldes begreifen (Parry 2004). Dies zielt keinesfalls auf eine Reetablierung eines Basis-Überbau-Modells und ebenso wenig auf eine in diesem Sinne reduktionistisches Verhältnis von ,materieller' und ,ideologischer' Ebene, sehr wohl aber darauf, sich nicht aufgrund der Komplexität und der Probleme, wie ich sie beispielsweise am Ideologiebegriff diskutiert habe (2.3.2.7), auf die Frage der Repräsentation zu beschränken. Dies hat auch Konsequenzen für Bildung. Dekoloniale politische Bildung müsste aus meiner Sicht nicht in erster Linie auf die 
Durchsetzung einer korrekteren Sprache zielen. Sprache ist ein wichtiger Schauplatz von Aushandlungen - ohne eine Kritik und Veränderung von Sprache wird es keinen epistemologischen Wandel geben. Dabei kann aber die Fokussierung auf Sprache auch dazu führen, dass über die Norm eines elaboriert-reflexiven Sprachgebrauchs Ausschluss und Distinktion anhand von Bildungszugängen hergestellt wird. Die Auflösung der Gegenüberstellung von Repräsentation und ,Materialität ‘ im poststrukturalistischen Sinne darf nicht dazu führen, dass die ,Materialität ' von Herrschaft bzw. Kolonialität ausgeblendet wird. Kolonialität umfasst nicht nur die Repräsentation der Welt, sondern auch die Struktur der sozialen Ungleichheit und damit letztlich auch der Frage der Vergesellschaftungsform selbst. Mit dem Ansatz der Kolonialität wird das Problem nicht einfach auf beiden Ebenen adressiert, sondern die ,materielle" und ,diskursive" Ebene werden als Einheit verstanden. Für die Frage von dekolonialer politischer Bildung bedeutet das aus meiner Sicht, dass Bildung gleichzeitig sowohl auf die Stärkung einer dekolonialen Reflexivität der eigenen Verstricktheit und Kompliz_innenschaft als auch auf die Stärkung von Handlungsfähigkeit als Intervention in gesellschaftliche Machtverhältnisse zielt.

Dabei sehe ich als Stärke meines Ansatzes an, dass der Fokus auf die subjektiven Sinngebungen der Schüler_innen es ermöglicht hat, Umgangsformen mit kolonialen und dekolonialen Erbschaften in ihren ambivalenten und widersprüchlichen Formen zu ko-konstruieren. Sowohl die zahlreichen kolonialen als auch die dekolonialen Momente, die meine Analyse sichtbar gemacht hat, unterstreichen zunächst die Notwendigkeit einer dekolonialen politischen Bildung und geben zwar keine eindeutigen Antworten auf die Frage der bildungspraktischen Umsetzung, konturieren jedoch Fragen und Probleme, die für die Praxis von Bedeutung sind. Ich halte es für sinnvoll, die zahlreichen Erfahrungen aus vergangenen und bestehenden dekolonialen Bildungs- und Forschungsprojekten dialogisch zusammen zu bringen, nicht um daraus den einen, ,richtigen ' Ansatz abzuleiten, sondern um voneinander lernen zu können. Gleichzeitig bin ich der Überzeugung, dass es weiterer empirischer Forschung bedarf, insbesondere auf der subjektbezogenen Ebene, um nicht nur die didaktischen Inhalte durch dekoloniale Momente zu ergänzen oder zu überschreiben, sondern auch ein Bildungsverständnis weiter zu vertiefen, in dem die Differenz von Lehrenden und Lernenden - und analog dazu von Fachwissenschaft zu Lernendenvorstellungen - aus einer dekolonialen Perspektive untergraben und anders gedacht werden kann (2.3). In diesem Sinne habe ich in meiner Forschung versucht, viel von den Schüler_innen zu lernen.

Karen Pashby macht darauf aufmerksam, dass die Übersetzung der theoretischen Arbeit in (schulische) Bildungspraxis komplex und „messy“ ist. „There is no purity in it, and it is about thinking much more strategically about social 
change and what we have learned is that change is going to happen anyway." (Pashby 2012: 23) Der Wirkungsgrad von institutionalisierten Bildungsprozessen sollte nicht überzeichnet werden. Institutionalisierte Bildungsprozesse sind eben nur ein Teil der Lebenswelt der Schüler_innen. Der stattfindende gesellschaftliche Wandel (change) betrifft aus meiner Sicht die koloniale Situation; dieser Wandel wird nicht von dekolonialen Bildungspraxen initiiert, sie kann höchstens zu seiner Stärkung beitragen. Dies wird auch durch die Ergebnisse meiner Analyse der Schüler_innenvorstellungen und der starken Präsenz kolonialer und dekolonialer Momente in ihren Vorstellungen untermauert. Will also Bildung dem Anspruch gerecht werden, (beispielsweise) Schüler_innen in die Lage zu versetzen, in ihrer eigenen Lebenswelt Reflexivität und Handlungsfähigkeit zu erlangen bzw. zu stärken, sollte die dekoloniale Option (Tlostanova/Mignolo 2012) zu einem fundamentalen Bestandteil der Didaktik der politischen Bildung werden. Angesichts einiger meiner Erfahrungen aus dem Erhebungskontext Schule heraus formuliert, könnte es die Aufgabe einer dekolonialen politischen Bildung sein, dazu beizutragen, dass Lernende dekoloniale Selbstreflexivität und Handlungsfähigkeit nicht trotz, sondern in der Folge von institutionalisierter Bildungspraxis erlangen bzw. stärken.

Wie jede Forschung ist diese Untersuchung durch eine partikulare Perspektive bestimmt. Dies habe ich versucht reflexiv zu bestimmen, indem ich sie als Spannung zwischen meiner Subjektposition und meiner Subjektpositionierung mehrfach diskutiert habe (2.3.3.5; 4.2.3.1.5). Meine Subjektposition bezieht sich mindestens auf meine Verortung in den vermachteten Differenzkategorien innerhalb rassistischer Verhältnisse, der internationalen Arbeitsteilung und der quasi feudalen Ordnung der globalen Geburtsrechtslotterie sowie den damit verbundenen Privilegien und die von dieser spezifischen Verortung verursachte Einschränkung der Sicht (4.2.4.3). Trotz der sozialen und politischen Verbundenheit mit vielen von Rassismus deprivilegierten Freund_innen und Familienmitgliedern ist diese Subjektposition nicht abzuschütteln, sondern bleibt vielmehr wirksam (4.2.3.1.5). Meine Subjektpositionierung bezieht sich auf mein Begehren und meine Praxis, diese Ungleichheiten ermöglichende Struktur infrage zu stellen. Meine Perspektive verstehe ich dabei als konstituiert durch das Spannungsverhältnis zwischen diesen beiden Polen, die nicht in die eine oder andere Richtung auflösbar ist. Meine von einer privilegierten Position aus stattfindenden Bildungsprozesse kann ich so als Auseinandersetzungen mit diesem Spannungsverhältnis verstehen, in denen sowohl mein Verhältnis zur Subjektposition als auch zur Subjektpositionierung immer wieder neu zu bestimmen ist. Das hier zugrunde liegende Konzept des Subjekts habe ich in Bezug auf das Erbe als ein brüchiges, nicht festes, kontingentes und nichtsdestotrotz in einem spezifischen Verhältnis zu den Machtverhältnissen stehendes Subjekt bestimmt (2.3). 
Angesichts der Partikularität meiner Perspektive ist weitere Forschung mit verwandten Fragestellungen, aber unter Einbezug anderer Perspektiven, möglicherweise auch in kollektiven, verschiedene Perspektiven zusammenbringenden Forschungszusammenhängen, aus meiner Sicht unbedingt anzustreben, um die hier vorliegende Perspektive durch andere Perspektiven zu ergänzen oder infrage zu stellen. Dabei halte ich den subjektzentrierten Ansatzes nach wie vor für zielführend, bei dem ein dekoloniales Erbe niemals als solches festgeschrieben werden kann, sondern sich immer wieder kontextuell, ausgehend von den Subjekten innerhalb von Bildungskonstellationen neu bestimmt. Insbesondere hoffe ich darauf, dass dekoloniale Perspektiven im Rahmen von politikdidaktischer Vorstellungsforschung (2.3.1) und Inclusive Citizenship Education (2.3.2) zu einem wesentlichen Bestandteil werden, aber auch, dass sich um den Ansatz der Dekolonialität herum, ein eigenständiges Feld empirischer Forschungspraxis ausbildet. Dazu würde ich gerne beitragen.

Als letzten Punkt möchte ich auf die Zeitdimension eingehen. Die Interviews wurden im Sommer 2013 geführt. Das heißt vor dem Eintritt einiger Ereignisse, die entscheidende von mir diskutierte Aspekte nachdrücklich geprägt haben. Anders ausgedrückt: Wahrscheinlich wären einige Ergebnisse heute anders ausgefallen. $\mathrm{Zu}$ nennen sind hier neben dem erwähnten Erstarken der Black Lives Matter-Bewegung nicht zuletzt der sogenannte "lange Sommer der Migration“ (Hess et al 2016), das in den Folgejahren als gesellschaftlicher Backlash zu bezeichnende Erstarken rechtspopulistischer Bewegungen, rassistische Diskursereignisse wie Silvester 2015 (Mecheril 2016) und die restriktiven, migrationspolitischen Gesetzesverschärfungen sowie die Kämpfe dagegen (Schwenken/Kirchhof 2016). Ebenfalls relevant, diskursprägend und damit möglicherweise Schüler_innenvorstellungen beeinflussend können auch Ereignisse wie die Debatten um den sogenannten „EU-Flüchtlingsdeal mit der Türkei“ (Gottschlich 2020) oder aber die Auseinandersetzungen um die Kriminalisierung von Rettungsaktionen von Geflüchteten auf dem Mittelmeer durch zivilgesellschaftliche Akteur_innen sein, die in der zwischenzeitlichen Verhaftung von Carola Rackete mündeten (Sea Watch 2020).

Es ließen sich weitere medial inszenierte Ereignisse anführen, die in zu späteren Zeitpunkten geführten Interviews wahrscheinlich eine Rolle gespielt hätten. Strukturell hat sich allerdings seitdem wenig geändert, woraus ich schließe, dass als wesentlich ausgemachte Denkweisen auch heute in ähnlicher Form ko-konstruiert werden würden. Der Verweis auf den Zeitkern aller Ergebnisse empirischer Vorstellungsforschung sehe ich dabei weniger als Infragestellung dieser Arbeit, sondern vielmehr als Stärkung des Forschungsdesiderats, also 
der Notwendigkeit weiterer dekolonial ausgerichteter und subjektzentrierter Forschung für die politische Bildung. Dies wäre eine Form unter anderen, das Ziel $\mathrm{zu}$ verfolgen, zu einer Dekolonisierung politischer Bildung beizutragen. Angesichts der hier aufgezeigten Präsenz kolonialer und dekolonialer Momente in den Schüler_innenvorstellungen erscheinen dekoloniale Perspektiven für die politische Bildung als unerlässlich. Wenn die subjektiven Vorstellungen zur gesellschaftlichen Orientierung und Handlungsfähigkeit der Schüler_innen Ausgangs- und Zielpunkt politischer Bildung sein sollen, braucht es dekoloniale Perspektiven. Dekoloniale politische Bildung ist aus meiner Sicht ein wichtiges Feld, um dekoloniale Perspektiven, Akteur_innen und Transformationsprozesse zu stärken - und insofern gerade vor dem Hintergrund der hier genannten, aber in der empirischen Untersuchung nicht erfassten Ereignisse dringlich und notwendig. Sorgen wir dafür, eine Form politischer Bildung zu betreiben, die dazu beiträgt, dass die Symbolik der in den Fluss geworfenen Edward Colston-Statue sich in eine nachhaltige, gesellschaftliche Strukturen grundlegend transformierende Praxis übersetzt.

Open Access Dieses Kapitel wird unter der Creative Commons Namensnennung 4.0 International Lizenz (http://creativecommons.org/licenses/by/4.0/deed.de) veröffentlicht, welche die Nutzung, Vervielfältigung, Bearbeitung, Verbreitung und Wiedergabe in jeglichem Medium und Format erlaubt, sofern Sie den/die ursprünglichen Autor(en) und die Quelle ordnungsgemäß nennen, einen Link zur Creative Commons Lizenz beifügen und angeben, ob Änderungen vorgenommen wurden.

Die in diesem Kapitel enthaltenen Bilder und sonstiges Drittmaterial unterliegen ebenfalls der genannten Creative Commons Lizenz, sofern sich aus der Abbildungslegende nichts anderes ergibt. Sofern das betreffende Material nicht unter der genannten Creative Commons Lizenz steht und die betreffende Handlung nicht nach gesetzlichen Vorschriften erlaubt ist, ist für die oben aufgeführten Weiterverwendungen des Materials die Einwilligung des jeweiligen Rechteinhabers einzuholen.

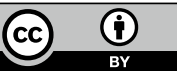

